\title{
Overexpression of Id-1 protein is a marker in colorectal cancer progression
}

\author{
ZENG-REN ZHAO $^{1 *}$, ZHI-YONG ZHANG ${ }^{2,3^{*}}$, HONG ZHANG $^{4}$, \\ LI JIANG ${ }^{3}$, MING-WEI WANG ${ }^{5}$ and XIAO-FENG SUN ${ }^{2}$ \\ ${ }^{1}$ Department of General Surgery, The First Hospital of Hebei Medical University, \\ Shijiazhuang 050031, P.R. China; ${ }^{2}$ Department of Oncology, Institute of Biomedicine and Surgery, \\ Linköping University, Linköping S-58185, Sweden; ${ }^{3}$ Department of Pathology, Tangshan Gongren Hospital, \\ Tangshan 063000, P.R. China; ${ }^{4}$ Department of Dermatology, Institute of Biomedicine and Surgery, \\ Linköping University, Linköping S-58185, Sweden; ${ }^{5}$ Laboratory Centre, The First Hospital \\ of Hebei Medical University, Shijiazhuang 050031, P.R. China
}

Received August 31, 2007; Accepted October 26, 2007

\begin{abstract}
The inhibitor of differentiation/DNA binding 1 (Id-1), a negative regulator of basic helix-loop-helix transcription factors, plays an important role in the regulation of cell proliferation and differentiation. We examined the Id-1 expression by immunohistochemistry in 9 adenomas, 79 primary colorectal adenocarcinomas matched with 40 adjacent normal mucosa specimens and its relationship with clinicopathological factors. The Id-1 expression was increased in the carcinoma compared to the adjacent normal mucosa either in the unmatched and matched samples or to the adenoma. There was no significant difference in the Id-1 expression between normal mucosa and adenoma. The Id-1 expression of carcinoma was increased from Dukes' stages A to $\mathrm{B}$, to $\mathrm{C}$ and to $\mathrm{D}$. The cases with lymph node metastasis had a higher rate of a stronger Id-1 expression than those without lymph node metastasis. In conclusion, Id-1 overexpression plays an important role in colorectal cancer progression.
\end{abstract}

\section{Introduction}

The helix-loop-helix (HLH) transcription factor has a highly conserved HLH structural domain consisting of two $\alpha$ helices separated by a loop region of variable sequences and length

Correspondence to: Dr Xiao-Feng Sun, Department of Oncology, Institute of Biomedicine and Surgery, Linköping University, Linköping S-581 85, Sweden

E-mail: xiasu@ibk.liu.se

*Contributed equally

Key words: inhibitor of differentiation/DNA binding, colorectal cancer, progression, immunohistochemistry
(1). The HLH structural motif of these proteins mediates dimerization and the $\mathrm{N}$-terminal basic domain is responsible for DNA binding and gene activation (1). Hererodimers regulate the expression of cell cycle regulatory proteins and tissue-specific genes formed between tissue-specific and ubiquitous basic HLH (bHLH) transcription factor binding to a so-called 'E-box' DNA sequence (2). The inhibitor of differentiation/DNA binding (Id), a member of the HLH family, functions by dimerization with other bHLH proteins.

Four members of the Id family, Id1-4, have already been identified and the genes are located on 20q11, 2p25, 1p36.1 and $6 \mathrm{p} 21-22$, respectively, which encode proteins ranging from 13 to $20 \mathrm{kDa}$. Id proteins act as dominant negative regulators of bHLH DNA binding proteins by preventing DNA binding and gene transcription (3). Id-1 has been reported as an oncogene that regulates cellular senescence, growth and survival in the development of tumors $(4,5)$. Moreover, Id-1 overexpression was associated with tumor invasion, advanced stage and poor prognosis in prostate (6), breast (7), endometrial (8) and cervical cancers (9). The abrogation of Id1-3 functions, by an antisense oligonucleotide blockade or by the enforced expression of the bHLH protein, led to growth arrest in human colon cancer cell lines (10).

Our study examined the Id-1 expression by immunohistochemistry in adjacent normal mucosa, adenoma and adenocarcinoma and investigated the correlation between the Id-1 expression and clinicopathological factors in cancer patients.

\section{Materials and methods}

Patients. Adenocarcinomas were collected from 79 patients with colorectal adenocarcinoma. Among them, 40 cases had adjacent normal mucosa, i.e., normal mucosa adjacent to primary adenocarcinoma on the same slides, histologically free from pre-tumor and tumor. The study also included 9 adenomas from additional patients with adenoma. The patients were diagnosed at the Department of Pathology, Tangshan Gongren Hospital, China, between 2004 and 2005. 
None of the patients received preoperative radiotherapy and chemotherapy. The patient gender, age, tumor site, size, gross status, invasive depth, growth pattern, Dukes' stage, lymph node status, histological type, grade of differentiation, necrosis, inflammatory reaction and fibrosis were obtained from surgical and pathological records. The mean age of cancer patients at the time of surgery was 58 years (range, 27-83 years). Right-sided tumors were found in 22 cases including caecum, ascending and transverse colon, left-sided ones were found in 16 cases including descending and sigmoid colon and rectal ones were found in 41 cases. The mean diameter of carcinomas was $4.7 \mathrm{~cm}$ (range, $1.5-13.0 \mathrm{~cm}$ ). The growth pattern was divided into expansive- or infiltrative-type based on the pattern of the tumor cell growth and invasiveness. Differentiation was graded as good, moderate and poor. All the slides including adenomas and adenocarcinomas along with adjacent normal mucosa were confirmed by two pathologists (Zhang ZY and Zhang $\mathrm{H}$ ).

Immunohistochemical staining. The sections $(5 \mu \mathrm{m})$ from paraffin-embedded tissue blocks were incubated at $60^{\circ} \mathrm{C}$ overnight, deparaffinized in xylene, re-hydrated in graded ethanol and washed in distilled water. To retrieve the masked epitope, the sections were boiled in $0.01 \mathrm{M}$ citrate buffer (pH 6.0) for $2 \mathrm{~min}$ in a high-pressure cooker. After cooling at room temperature for $30 \mathrm{~min}$ and washing in phosphatebuffered saline (PBS, pH 7.4) buffer, the endogenous peroxidase was blocked with $0.3 \% \mathrm{H}_{2} \mathrm{O}_{2}$ containing methanol for $20 \mathrm{~min}$ and rinsed with PBS. Then, the sections were incubated overnight at $4^{\circ} \mathrm{C}$ with a polyclonal rabbit antibody against Id-1 (Santa Cruz Biotechnology, Santa Cruz, CA) and diluted at 1:50 with PBS. After washing with PBS, the slides were incubated with polymer enhancer for $20 \mathrm{~min}$ at $37^{\circ} \mathrm{C}$ and then with polymerized horseradish peroxidase antimouse/rabbit IgG for 30 min [Elivision ${ }^{\mathrm{TM}}$ plus polyer horseradish peroxidase (mouse/rabbit) IHC kit, Fuzhou Maixin Biology Technology Limited Company, Fuzhou, China]. The peroxidase reaction was performed by the use of 3,3'-diaminobenzididine for $2 \mathrm{~min}$. The sections were finally counterstained with haematoxylin and mounted.

The sections known to stain positively were included as negative and positive controls. For negative controls, the sections incubated with PBS instead of the primary antibody were not stained, unlike the positive controls.

The slides were microscopically examined and scored independently by the two pathologists without any clinical or histopathological information. To avoid artefacts, areas with poor morphology, margins of the sections and necrosis were not considered. The Id-1 expression was predominantly present in the cytoplasm of tumor and normal epithelial cells, as well as fibroblast and muscles of blood vessels in the tumorassociated stroma. Thus, the Id-1 expression in the tumor and epithelial cells, and stroma was respectively classified as negative, weak, moderate or strong. In the sections showing a heterogeneous staining pattern, if the stronger staining was more than one third of the sections the staining was considered for scoring. Among the 128 sections evaluated, there were discountenances in 22 sections in the first round of the evaluation. These sections were re-read by the two pathologists, individually and matched. The final 6 discrepant sections were re-examined by dual-microscope and a concurrent score was achieved.

Statistical analysis. The Chi-square test, Fisher exact test or McNemar's method was used to test the relationship of the Id-1 expression in normal mucosa, adenoma and carcinoma, as well as the relationship between the Id-1 expression and clinicopathological variables. Spearman's coefficient of correlation was used to test the correlation between the Id-1 expression in tumor and stroma. All P-values mentioned are two-sided and P-values $<0.05$ were judged as statistically significant.

\section{Results}

The Id-1 expression in adjacent normal mucosa, adenoma and carcinoma. The Id-1 expression was examined in 40 adjacent normal mucosa samples, 9 adenomas and 79 carcinomas and was predominantly present in the cytoplasm of epithelial cells of normal mucosa and tumor cells (Fig. 1). Only 2 carcinomas showed weak nuclear staining, besides cytoplasmic staining. A strong Id-1 expression was also seen in tumor embolus in the vessels (Fig. 1). Fig. 2 presents the frequency of the Id-1 expression in normal mucosa, adenoma and carcinoma. In the normal mucosa samples, 1 (2.5\%) sample showed strong staining, $10(25.0 \%)$ showed moderate, $19(47.5 \%)$ showed weak and $10(25.0 \%)$ were negative. In the adenomas, $1(11.1 \%)$ case had strong staining, 2 (22.2\%) had moderate and $6(66.7 \%)$ had a weak expression. In the carcinomas, $23(29.1 \%)$ had strong, $43(54.4 \%)$ had moderate and $13(16.5 \%)$ had weak staining. There was no negative case in the adenomas and carcinomas.

According to the similarity of clinicopathological features, in further statistical analyses, cases with an Id-1 negative and weak expression were considered as a weaker staining group while moderate and strong-stained cases as a stronger staining group. The Id-1 stronger expression was significantly increased in carcinomas when compared to the adjacent normal mucosa either in the unmatched cases $(\mathrm{P}<0.0001)$ or matched cases $(\mathrm{P}<0.0001)$ and was also significantly increased in carcinomas when compared to adenomas $(\mathrm{P}=0.003)$. However, there was no significant difference of the expression between the adjacent normal mucosa and adenoma $(\mathrm{P}=0.73)$.

The Id-1 expression in carcinomas in relation to clinicopathological variables. We analyzed the relationship between the Id-1 expression in carcinomas and clinicopathological variables. There was a significant difference of the Id-1 expression among Dukes' stages $(\mathrm{P}=0.03$, Table I). Further analysis showed that the frequency of the Id-1 stronger expression was significantly increased from Dukes' A to $\mathrm{C}(50.0 \%$ vs. $88.5 \%, \mathrm{P}=0.04)$ and tended to be increased from $\mathrm{A}$ to $\mathrm{D}(50.0 \%$ vs. $100.0 \%, \mathrm{P}=0.07)$. There were no differences between Dukes' stages A and B (50.0\% vs. $71.4 \%, \mathrm{P}=0.61), \mathrm{B}$ and $\mathrm{C}(71.4 \%$ vs. $88.5 \%, \mathrm{P}=0.11)$, as well as $\mathrm{C}$ and $\mathrm{D}(\mathrm{P}=0.15)$. The frequency of the Id-1 stronger expression was higher in carcinomas with lymph node metastasis compared to those without metastasis $(89.5 \%$ vs. $68.2 \%, \mathrm{P}=0.02$, Table I). There was no significant association 


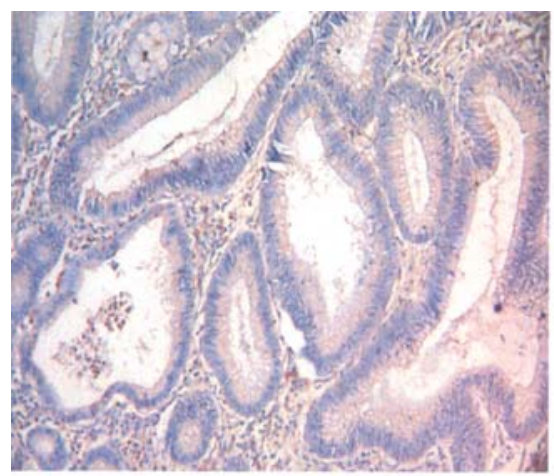

A

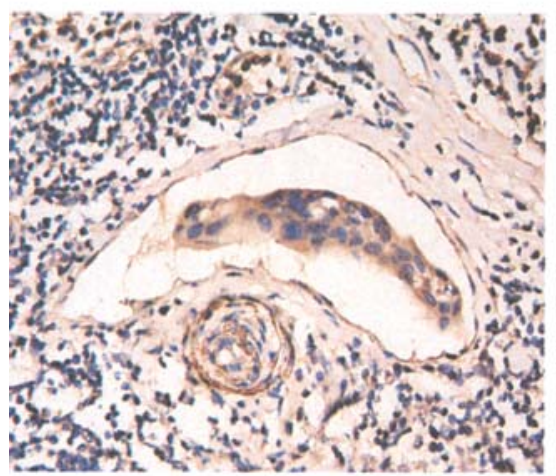

C

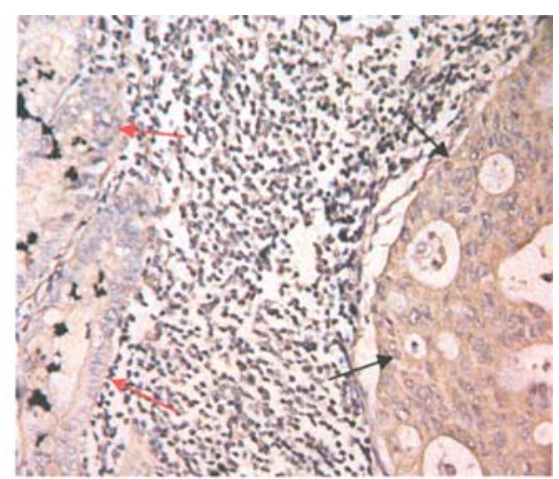

B

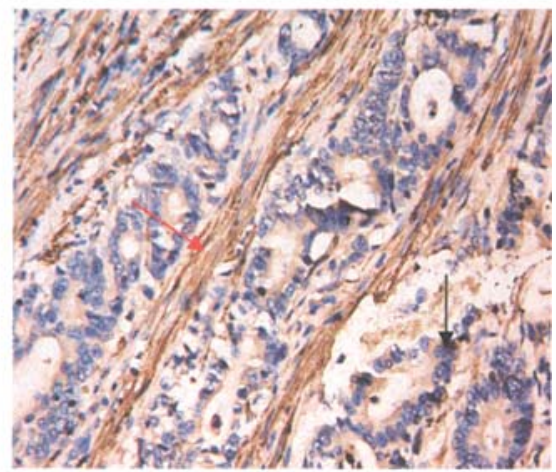

D

Figure 1. Id-1 immunohistochemistry in adjacent normal mucosa, adenoma and adenocarcinoma of colorectum. (A) Weak expression in adenoma. (B) A weak expression in adjacent normal mucosa (red arrow) and a strong expression in carcinoma (black arrow). (C) Strong expression in tumor embolus. (D) A strong expression in stroma (red arrow) and weak in carcinoma (black arrow).

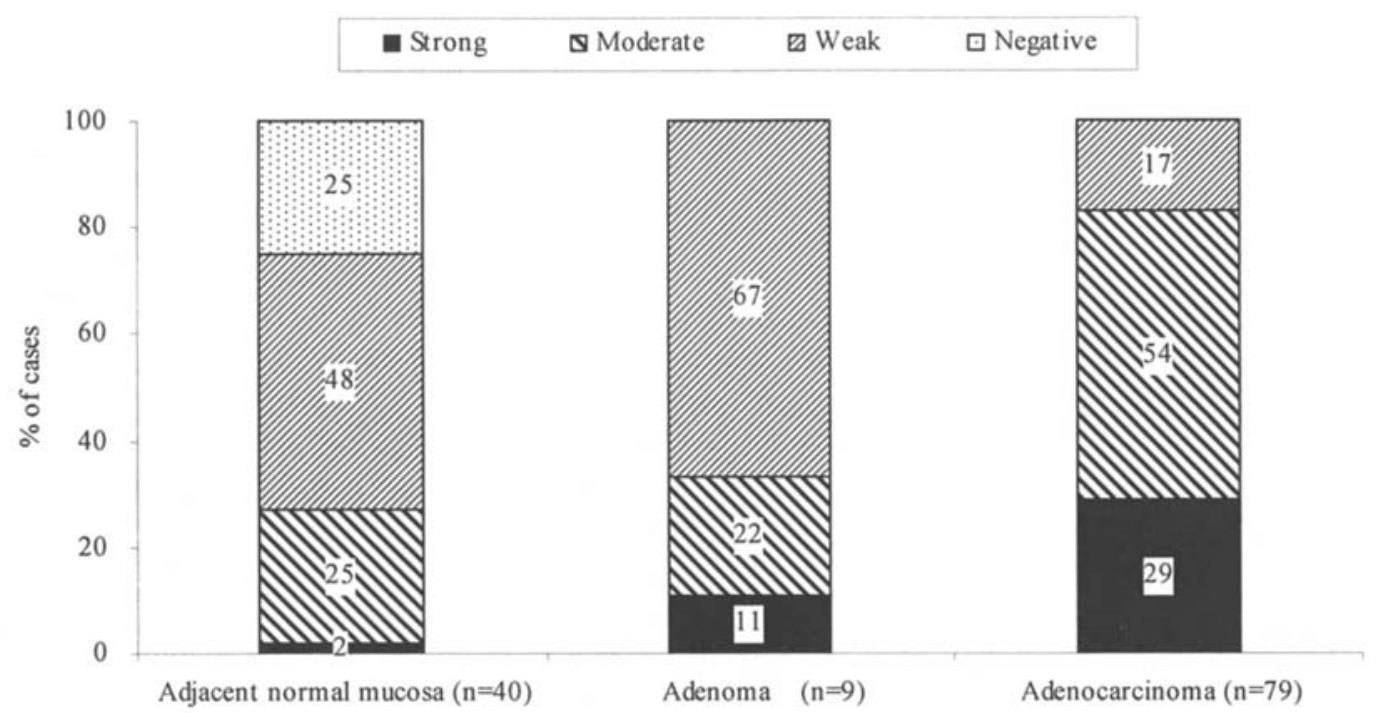

Figure 2. The Id-1 expression in adjacent normal mucosa, adenoma and adenocarcinoma of the colorectum.

of the Id-1 expression with other clinicopathological variables including gender, age, tumor site, size, gross status, invasive depth, growth pattern, histological type, grade of differentiation, necrosis, inflammatory reaction and fibrosis $(\mathrm{P}>0.05$, Table I).

Id-1 was also present in the cytoplasm of the fibroblast and the muscle of the blood vessels in the tumor-associated stroma (Fig. 1) and there were $60(75.9 \%)$ cases in the stronger group (including 27 moderate staining) and $19(24.1 \%)$ in the weaker group (including 1 negative case). We further examined the Id-1 expression in the stroma of carcinomas in relation to clinicopathological variables and found that men had a higher rate of the Id-1 stronger expression than women $(85.7 \%$ vs. $64.9 \%, P=0.03)$. There was no significant 
Table I. The Id-1 expression in relation to clinicopathological variables in colorectal adenocarcinoma.

\begin{tabular}{|c|c|c|c|c|c|c|}
\hline \multirow{2}{*}{ Variables } & \multirow{2}{*}{$\mathrm{n}$} & \multicolumn{2}{|c|}{ Weaker } & \multicolumn{2}{|c|}{ Stronger } & \multirow{2}{*}{ P-value } \\
\hline & & $\mathrm{n}$ & $(\%)$ & $\mathrm{n}$ & $(\%)$ & \\
\hline Gender & & & & & & 0.58 \\
\hline Men & 42 & 6 & (14.3) & 36 & $(85.7)$ & \\
\hline Women & 37 & 7 & $(18.9)$ & 30 & (81.1) & \\
\hline Age (years) & & & & & & 0.80 \\
\hline$\leq 58$ & 40 & 7 & $(17.5)$ & 33 & $(82.5)$ & \\
\hline$>58$ & 39 & 6 & $(15.4)$ & 33 & $(84.6)$ & \\
\hline Tumor site & & & & & & 0.40 \\
\hline Right & 22 & 5 & $(22.7)$ & 17 & (77.3) & \\
\hline Left & 16 & 1 & $(6.3)$ & 15 & (93.7) & \\
\hline Rectum & 41 & 7 & $(17.1)$ & 34 & $(82.9)$ & \\
\hline Tumor size $(\mathrm{cm})$ & & & & & & 0.28 \\
\hline$\leq 4.7$ & 44 & 9 & $(20.5)$ & 35 & (79.5) & \\
\hline$>4.7$ & 35 & 4 & $(11.4)$ & 31 & (88.6) & \\
\hline Gross status & & & & & & 0.72 \\
\hline Polyploid & 15 & 2 & $(13.3)$ & 13 & (86.6) & \\
\hline Ulcerative & 64 & 11 & $(17.2)$ & 53 & $(82.8)$ & \\
\hline Invasive depth & & & & & & 0.35 \\
\hline Intra-bowel wall & 13 & 1 & $(7.7)$ & 12 & (92.3) & \\
\hline Ultra-bowel wall & 66 & 12 & $(18.2)$ & 54 & $(81.8)$ & \\
\hline Growth pattern & & & & & & 0.73 \\
\hline Expansive & 33 & 6 & $(18.2)$ & 27 & (81.8) & \\
\hline Infiltrative & 46 & 7 & $(15.2)$ & 39 & $(84.8)$ & \\
\hline Dukes' stage & & & & & & 0.03 \\
\hline A & 6 & 3 & $(50.0)$ & 3 & $(50.0)$ & \\
\hline B & 14 & 4 & $(28.6)$ & 10 & (71.4) & \\
\hline $\mathrm{C}$ & 52 & 6 & $(11.5)$ & 46 & $(88.5)$ & \\
\hline $\mathrm{D}$ & 7 & 0 & $(0.0)$ & 7 & $(100.0)$ & \\
\hline Lymph node status & & & & & & 0.02 \\
\hline Non-metastasis & 22 & 7 & $(31.8)$ & 15 & $(68.2)$ & \\
\hline Metastasis & 57 & 6 & $(10.5)$ & 51 & $(89.5)$ & \\
\hline Histological type & & & & & & 0.16 \\
\hline Non-mucinous & 61 & 12 & $(19.7)$ & 49 & $(80.3)$ & \\
\hline Mucinous/signet-ring cell & 18 & 1 & $(5.6)$ & 17 & $(94.4)$ & \\
\hline Grade of differentiation & & & & & & 0.95 \\
\hline Good & 17 & 3 & $(17.6)$ & 14 & (82.3) & \\
\hline Moderate & 54 & 9 & $(16.7)$ & 45 & (83.3) & \\
\hline Poor & 8 & 1 & $(12.5)$ & 7 & $(87.5)$ & \\
\hline Necrosis & & & & & & 0.26 \\
\hline$\leq 30 \%$ & 73 & 13 & $(17.8)$ & 60 & $(82.2)$ & \\
\hline$>30 \%$ & 6 & 0 & $(0.0)$ & 6 & $(100.0)$ & \\
\hline Inflammatory reaction & & & & & & 0.63 \\
\hline Weak & 63 & 11 & $(17.5)$ & 52 & $(82.5)$ & \\
\hline Strong & 16 & 2 & $(12.5)$ & 14 & $(87.5)$ & \\
\hline
\end{tabular}


Table I. Continued.

\begin{tabular}{|c|c|c|c|c|c|c|}
\hline \multirow{2}{*}{ Variables } & \multirow{2}{*}{$\mathrm{n}$} & \multicolumn{2}{|c|}{ Weaker } & \multicolumn{2}{|c|}{ Stronger } & \multirow{2}{*}{ P-value } \\
\hline & & $\mathrm{n}$ & $(\%)$ & $\mathrm{n}$ & $(\%)$ & \\
\hline Fibrosis & & & & & & 0.79 \\
\hline Weak & 46 & 8 & (17.4) & 38 & (82.6) & \\
\hline Strong & 33 & 5 & (15.2) & 28 & (84.8) & \\
\hline
\end{tabular}

${ }^{\mathrm{a} C h i}$-square or Fisher exact test.

association of the Id-1 expression in the stroma with other clinicopathological variables ( $P>0.05$, data not shown). Spearman's coefficient of correlation revealed a significant correlation of the Id-1 expression between the tumor cells and stroma $(\mathrm{r}=0.39 ; \mathrm{P}=0.0004)$.

\section{Discussion}

The Id-1 expression was up-regulated in several types of human cancers including colorectal (10), gastric (11), ovarian (12) and medullary thyroid cancer (13) when compared to the corresponding normal tissues. In the present study, the Id-1 expression was significantly increased in colorectal carcinoma compared to the adjacent normal mucosa or the adenoma. These results suggest that Id-1 played a role in the development of tumors. Moreover, we found that the adenoma did not differ from normal mucosa as regards the Id-1 expression, which was in line with a previous study where the Id-1 expression did not increase in the adenoma compared to the normal mucosa in the colorectum (10). Together with this study, we propose that the increased expression of Id-1 may be a relatively late event during colorectal tumorigenesis. However, the expression of Id-1 was up-regulated in C-cell hyperplastic foci in the medullary thyroid cancer (13), dysplastic/metaplatic ducts in chronic pancreatitis (14) and cirrhosis of the liver with a high risk of hepatocellular carcinoma development (15). These results indicated that Id-1 may be an early marker of pancreatic, thyroid and hepatocellular malignant transformation (13-15). These inconsistent results may be partly due to the different types of tumors.

Id- 1 has been found to be involved in the invasion and metastasis of gastric (11), oral (16), breast (17) and prostate cancers (18). In the present study, the frequency of the Id-1 stronger expression was significantly increased in the advanced Dukes' stage and the cases with lymph node metastasis. However, Wilson et al (10) did not find an association of Id-1 with Jass stages in colorectal cancer although they found a relationship of the Id-2 expression with the advanced Jass stage. This may depend on the stage system, number of cases, etc. As a negative regulator of transcription, Id-1 may be responsible for changes in gene expression that led to an increased growth and invasion of tumor cells. The constitutive expression of Id-1 converted the non-aggressive prostate cancer cells into more proliferative, invasive and migratory phenotype cells (18). Human metastastic breast cancer cells became significantly less invasive in vitro and less metastasis in vivo after a down-regulated Id-1 expression.
A systemically targeted Id-1 expression in vivo may reduce metastases of breast cancer cells in tumor-bearing mice (17). The evidence supports our results that the Id- 1 protein was involved in tumor invasion and metastasis and may play a pivotal role in the progression of colorectal cancer.

Tumor invasion is a multi-step process and many of the stages require degradation of the extracellular matrix (ECM) (19). Matrix metalloproteinases (MMPs) are a major family regulating the degradation of the basement membrane and ECM. The overexpression of MMPs was involved in tumor initiation, invasion and metastasis. During the involution of the mammary gland, the Id-1 expression resulted in the upregulation of a novel $120 \mathrm{kDa}$ MMPs protein, a type IV collagenase MMP family member and directly correlated with the invasiveness of breast cancer cell lines and thus degraded gelatine (20). Breast cancer cells transfected with Id-1 dissociated and invaded the basement membrane and resumed proliferation (20). The high expression of Id-1 also induced an increased secretion of MMP-2 in prostate cancer (18). In the present study, we first examined the Id-1 expression in the stroma and further found that the stromal Id-1 expression was significantly correlated with the tumor cellular Id-1 expression. It may be that there was an interaction between the stromal and tumor cellular Id-1 in tumor progress. We could not prove whether Id-1 was involved in tumor progression by interacting with ECM, especially through the up-regulation of MMPs.

We found that male patients had a higher rate of a stronger Id-1 expression in the stroma than female patients. Epidemiological and molecular studies showed a gender difference in several aspects. For example, a higher incidence was seen in males but proximal cancers were more common in females. A reduced risk of colon cancer was demonstrated with the use of hormone replace treatment (21). Moreover, the effect of the sex steroid on cell growth could be partially substituted or stimulated by the Id-1 expression in breast and prostate cancer cell lines $(7,22)$. Id-1 may contribute to the regulation of multiple pathways for hormone stages in colorectal cancer.

In conclusion, our results suggest that Id-1 protein was involved in the development of colorectal cancers and its overexpression may be a marker in tumor progression.

\section{Acknowledgements}

The study was supported by grants from the Service Centre for Experts and Scholars of Hebei Province, China, the Swedish Cancer Foundation and the Health Research Council of the 
South-East of Sweden. The authors also thank Hai-Xian Jiang for collecting patient information and establishing the database.

\section{References}

1. Kadesch T: Consequences of heteromeric interactions among helix-loop-helix proteins. Cell Growth Differ 4: 49-55, 1993.

2. Norton JD, Deed RW, Craggs G and Sablitzky F: Id helix-loophelix proteins in cell growth and differentiation. Trends Cell Biol 8: 58-65, 1998.

3. Benezra R, Davis RL, Lockshon D, Turner DL and Weintraub H: The protein Id: a negative regulator of helix-loop-helix DNA binding proteins. Cell 61: 49-59, 1990.

4. Nickoloff BJ, Chaturvedi V, Bacon P, Qin JZ, Denning MF and Diaz MO: Id-1 delays senescence but does not immortalize keratinocytes. J Biol Chem 275: 27501-27504, 2000.

5. Wang X, Xu K, Ling MT, Wong YC, Feng HC, Nicholls J and Tsao SW: Evidence of increased Id-1 expression and its role in cell proliferation in nasopharyngeal carcinoma cells. Mol Carcinog 35: 42-49, 2002.

6. Ouyang XS, Wang X, Lee DT, Tsao SW and Wong YC: Overexpression of Id-1 in prostate cancer. J Urol 167: 2598-2602, 2002.

7. Lin CQ, Singh J, Murata K, et al: A role for Id-1 in the aggressive phenotype and steroid hormone response of human breast cancer cells. Cancer Res 60: 1332-1340, 2000.

8. Takai N, Miyazaki T, Fujisawa K, Nasu K and Miyakawa I: Id-1 expression is associated with histogical grade and invasive behaviour in endometrial carcinoma. Cancer Lett 165: 185-193, 2001.

9. Schindl M, Oberhuber G, Obermair A, Schoppmann SF, Karner B and Birner P: Overexpression of Id-1 protein is a marker for unfavorable prognosis in early-stage cervical cancer. Cancer Res 61: 5703-5706, 2001.

10. Wilson JW, Deed RW, Inoue T, et al: Expression of Id helixloop-helix proteins in colorectal adenocarcinoma correlates with p53 expression and mitotic index. Cancer Res 61: 8803-8810, 2001.

11. Jang TJ, Jung KH and Choi EA: Id-1 gene downregulation by sulindac sulfide and its upregulation during tumor development in gastric cancer. Int J Cancer 118: 1356-1363, 2006.
12. Schindl M, Schoppmann SF, Strobel T, Heinzl H, Leisser C, Horvat R and Birner P: Level of Id-1 protein expression correlates with poor differentiation, enhanced malignant potential, and more aggressive clinical behavior of epithelial ovarian tumors. Clin Cancer Res 9: 779-785, 2003.

13. Kebebew E, Treseler PA, Duh QY and Clark OH: The helixloop-helix transcription factor, Id-1, is overexpressed in medullary thyroid cancer. Surgery 128: 952-957, 2000.

14. Maruyama H, Kleeff J, Wildi S, Friess H, Buchler MW, Israel MA and Korc M: Id-1 and Id-2 are overexpressed in pancreatic cancer and in dysphasic lesions in chronic pancreatitis. Am J Pathol 155: 815-822, 1999.

15. Matsuda Y, Yamagiwa S, Takamura M, Honda Y, Ishimoto Y, Ichida T and Aoyagi Y: Overexpressed Id-1 is associated with a high risk of hepatocellular carcinoma development in patients with cirrhosis without transcriptional repression of p16. Cancer 104: 1037-1044, 2005.

16. Nishimine M, Nakamura M, Mishima K, Kishi M, Kirita T, Sugimura M and Konishi N: Id proteins are overexpressed in human oral squamous cell carcinomas. J Oral Pathol Med 32: 350-357, 2003.

17. Fong S, Itahana Y, Sumida T, et al: Id-1 as a molecular target in therapy for breast cancer cell invasion and metastasis. Proc Natl Acad Sci USA 100: 13543-13548, 2003.

18. Coppe JP, Itahana Y, Moore DH, Bennington JL and Desprez PY: Id-1 and Id-2 proteins as molecular markers for human prostate cancer progression. Clin Cancer Res 10: 2044-2051, 2004.

19. Egeblad $M$ and Werb Z: New functions for the matrix metalloproteinases in cancer progression. Nat Rev Cancer 2: 161-174, 2002.

20. Desprez PY, Lin CQ, Thomasset N, Sympson CJ, Bissell MJ and Campisi J: A novel pathway for mammary epithelial cell invasion induced by the helix-loop-helix protein Id-1. Mol Cell Biol 18: 4577-4588, 1998.

21. International menopause society expert workshop: Hormone replacement therapy and cancer. Gynecol Endocrinol 15: 453-465, 2001.

22. Ling MT, Wang X, Ouyang XS, Xu K, Tsao SW and Wong YC: Id-1 expression promotes cell survival through activation of NFkappa B, signaling pathway in prostate cancer cells. Oncogene 22: 4498-4508, 2003 . 